\title{
SINGULARITIES OF THE RADON TRANSFORM
}

\author{
A. G. RAMM AND A. I. ZASLAVSKY
}

\begin{abstract}
Singularities of the Radon transform of a piecewise smooth function $f(x), x \in R^{n}, n \geq 2$, are calculated. If the singularities of the Radon transform are known, then the equations of the surfaces of discontinuity of $f(x)$ are calculated by applying the Legendre transform to the functions, which appear in the equations of the discontinuity surfaces of the Radon transform of $f(x)$; examples are given. Numerical aspects of the problem of finding discontinuities of $f(x)$, given the discontinuities of its Radon transform, are discussed.
\end{abstract}

\section{INTRODUCTION}

Let $f(x)$ be a compactly supported function, $D$ be its support, and $\Gamma=\partial D$ be a union of finitely many $C^{\infty}$ hypersurfaces $\Gamma_{1}, \ldots, \Gamma_{s}$ in general position, each of which can be written in local coordinates as

$$
x_{n}=g\left(x^{\prime}\right), \quad x^{\prime}=\left(x_{1}, \ldots, x_{n-1}\right), n \geq 2,
$$

where $g\left(x^{\prime}\right) \in C^{\infty}, f(x) \in C^{\infty}(D),\left.f(x)\right|_{\Gamma} \geq c>0$. The discontinuity surface of $f(x)$ is $\Gamma$, the boundary of $D$. We assume that the rank of the Hessian $g_{i j}(x):=\partial^{2} g / \partial x_{i} \partial x_{j}$ is constant on each of $\Gamma_{j}, 1 \leq j \leq s$.

Define the Radon transform (RT) of $f(x)$ by the usual formula [GGV] $\hat{f}(p, \alpha)=\int_{\mathbb{R}^{n}} f(x) \delta(p-\alpha \cdot x) d x$, where $\delta$ is the delta-function. It is well known that $\hat{f}(\lambda p, \lambda \alpha)=|\lambda|^{-1} \hat{f}(p, \alpha), \lambda \in R^{1}, \lambda \neq 0$. Consider the integral

$$
R(p, \alpha ; f):=\int_{l_{\alpha p}} f(x) \mu(d x),
$$

where $l_{\alpha p}$ is the plane $\alpha \cdot x-p=0, \alpha \in \mathbb{R}^{n}, p \in \mathbb{R}^{1}$, and $\mu(d x)$ is the Lebesgue measure on $l_{\alpha p}$. One has $R(p, \alpha ; f)=\hat{f}\left(p /|\alpha|, \alpha^{0}\right), \alpha^{0}:=\alpha|\alpha|^{-1}$, so that $R(p, \alpha ; f)=|\alpha| \hat{f}(p, \alpha),|\alpha|=\left(\alpha_{1}^{2}+\cdots+\alpha_{n}^{2}\right)^{1 / 2}$.

The problems we are interested in are: (P1) Find the singularities of $R(p, \alpha ; f)$; and (P2) Find the surface $\Gamma$ of discontinuity of $f(x)$ given the singularities of $R(p, \alpha ; f)$.

No results concerning (P2) were known. In $[\mathrm{N}]$ one can find an estimate of the norm of $\hat{f}(p, \alpha)$ in Sobolev spaces. This result does not give information about (P1) and (P2). In [P] there is a result given without proof, which has a relation to $(\mathrm{P} 1)$. Our result is more general. In $[Q]$ it is mentioned that the values $(\alpha: p)$, such that $l_{\alpha p}$ is tangent to $\Gamma$, play a special role. This observation is made quantitative in our Theorem 1 . Our results are useful for inversion of incomplete tomographic data $[R 2]$.

Received by the editors March 11, 1992.

1991 Mathematics Subject Classification. Primary 44A12. 
The basic results are formulated in §II. They give solutions of the problems (P1) and (P2). Actually, more general problems are solved; particularly, finite smoothness of $f(x)$ and $\Gamma$ is allowed, the role of the intersections of $\Gamma_{j}$ in the study of the singularities of $R(p, \alpha ; f)$ is clarified, etc. In §III proofs are sketched. In $\S I V$ examples are given. In $\S \mathrm{V}$ numerical aspects of problem (P2) are discussed.

We conclude this introduction by an outline of our ideas. First, we describe the behavior of $R(p, \alpha ; f)$ in a neighborhood of the set $Q_{f}$ which is the set of singularities of $R(p, \alpha ; f)$. Second, we prove that, in general, there is an equation of the set $Q_{f}$ which is of the form $q=h(\beta), \beta \in R^{n-1}$, so that $Q_{f}$ is a hypersurface. Third, we prove that the function $g\left(x^{\prime}\right)$ (in the equation of $\Gamma$ ) is the Legendre transform of the function $h(\beta)$ (in the equation of $Q_{f}$ ). Fourth, we describe some geometric properties of $Q_{f}$.

Our results give a theoretical basis for the solution of the practically important problem in nondestructive evaluation and remote sensing, the problem of finding the discontinuities of a function from the knowledge of its RT.

\section{FORMULATION OF THE RESULTS}

The RT, defined by formula (1), is a function on the projective space $\mathbb{R P}_{n}$, and we take $R(1,0 ; f):=0$ for compactly supported $f$. Let $Q_{f}$ denote the set of the points $(\alpha: p)$ in this projective space, which correspond to the planes $l_{\alpha p}$ tangent to $\Gamma=\partial D$. We say that $l_{\alpha p}$ is tangent to $\Gamma$ at a point $x \in B_{m}:=\bigcap_{j=1}^{m} \Gamma_{j}$, if $l_{\alpha p}$ is not transversal to $B_{m}$ at the point $x$.

1. Our first result is the following theorem in which the description of the singularities of $R(p, \alpha ; f)$ is given.

Let $l_{\alpha p}$ be tangent to $\Gamma$ at the point $\bar{x}$. We claim that if $\bar{\alpha}$ is generic, then the set $Q_{f}$ is a smooth hypersurface in a neighborhood $U$ of $(\bar{\alpha}: \bar{p})$. If $A$ is a symmetric matrix with real-valued entries, then its inertia index (inerdex) is defined to be the number of its negative eigenvalues. Consider first the case when $\Gamma$ consists of one surface.

Theorem 1. There exists an equation $\zeta(\alpha: p)=0, \nabla \zeta \neq 0$ in $U$, which defines $Q_{f}$ in $U$, and two $C^{\infty}$ functions $r_{1}$ and $r_{2}$ in $U$ such that

$$
R(p, \alpha ; f)= \begin{cases}\zeta_{+}^{(n-1) / 2} r_{1}+r_{2}, & \text { if } \text { In is even, } \\ \zeta^{(n-1) / 2}(\ln |\zeta|) r_{1}+r_{2}, & \text { if } \text { In is odd. }\end{cases}
$$

Here I is the inerdex of the matrix $z_{k j}$, where $z_{k j}$ is the Hessian of the function $z=(\bar{\alpha} \cdot x-\bar{p}) /|\bar{\alpha}|$ on $\Gamma$ at the point $\bar{x}$ and $z_{+}=\max (z, 0)$.

If $\bar{x} \in B_{m}$ and $(\bar{\alpha}: \bar{p})$ is generic, then the following result holds.

Theorem $\mathbf{1}^{\prime}$. There exists $\zeta(\alpha: p), \nabla \zeta \neq 0$ in $U$, such that the equation $\zeta(\alpha: p)=0$ is the equation of $Q_{f}$ in $U$, and two $C^{\infty}$ functions $r_{1}$ and $r_{2}$ in $U$, such that

$$
R(p, \alpha ; f)= \begin{cases}\zeta_{+}^{(n+m-2) / 2} r_{1}+r_{2}, & \text { if } I(n+m-1) \text { is even, } \\ \zeta^{(n+m-2) / 2}(\ln |\zeta|) r_{1}+r_{2}, & \text { if } I(n+m-1) \text { is odd. }\end{cases}
$$

In [RZ1] the constant $r_{1}(\bar{\alpha}: \bar{p})$ is calculated. In [RZ2] this result is used for a derivation of the asymptotics of the Fourier transform of a piecewise smooth function. 
2. Let us define the Legendre transform of a function $g(y), y \in R^{n-1}$ in a neighborhood $U_{\bar{y}}$ of a point $\bar{y}$ at which the matrix $g_{i j}(y):=\partial^{2} g / \partial y_{i} \partial y_{j}$ is nondegenerate, i.e., det $g_{i j}(y) \neq 0$ in $U_{\bar{y}}$. Define $L g:=h(\beta):=\beta \cdot y-g(y)$, where the dot stands for the inner product and $y=y(\beta)$ is the unique solution of the equation $\beta=\nabla g(y)$ in a neighborhood $U_{\bar{\beta}}$ of the point $\bar{\beta}=\nabla g(\bar{y})$. One can prove that if $g \in C^{l}\left(U_{\bar{y}}\right), l \geq 2$, and $\operatorname{det} g_{i j}(y) \neq 0$ in $U_{\bar{y}}$, then $h(\beta) \in C^{l}\left(U_{\bar{\beta}}\right)$.

It is known that under our assumptions $L h=g(y)$, i.e., the Legendre transform is involutive: $g(y)=\beta \cdot y-h(\beta)$, where $\beta=\beta(y)$ is the unique solution to the equation $y=\nabla h(\beta), \beta \in U_{\bar{\beta}}$. One can prove that $\operatorname{det} h_{i j}(\beta) \neq 0$ in $U_{\bar{\beta}}$ if $\operatorname{det} g_{i j}(y) \neq 0$ in $U_{\bar{y}}$; moreover, the matrix $h_{i j}(\beta)$ is inverse to $g_{i j}(y)$, where $\beta=\beta(y)$. Recall that $\Gamma$ is a union of hypersurfaces $\Gamma_{j}, 1 \leq j \leq s$, $\Gamma_{1}, \ldots, \Gamma_{s}$ are $C^{\infty}$ and in general position. Denote $\widehat{B}_{m}:=\Gamma_{1, \ldots, m}$ the set of $(\alpha: p) \in \mathbb{R P}_{n}$ such that $l_{\alpha p}$ is tangent to $B_{m}$. The set $\widehat{B}_{m} \subset \mathbb{R} \mathbb{P}_{n}$ may not be a hypersurface (see Theorem 3); however, as Theorem $1^{\prime}$ claims, it is indeed a smooth hypersurface outside a set of $(n-1)$-dimensional Lebesgue's measure zero.

3. Our second result gives the relation between the discontinuity surfaces for $R(p, \alpha ; f)$ and those for $f(x)$; namely, the function $g\left(x^{\prime}\right)$ in the local equation of $\Gamma, x_{n}=g\left(x^{\prime}\right)$, is the Legendre transform of the function $h(\beta)$ which gives the equation of $Q_{f}, q=h(\beta)$.

Assume that $q=h(\beta), \beta \in U_{\bar{\beta}}$, where $U_{\bar{\beta}}$ is a neighborhood of a point $\bar{\beta}, \bar{q}=h(\bar{\beta})$, and $\operatorname{det} h_{i j}(\beta) \neq 0$ in $U_{\bar{\beta}}$, where $h_{i j}:=\partial^{2} h / \partial \beta_{i} \partial \beta_{j}$. Let $\bar{x}^{\prime}=\nabla h(\bar{\beta})$.

Theorem 2. If $h(\beta) \in C^{l}\left(U_{\bar{\beta}}\right), l \geq 2$, then $L h=g\left(x^{\prime}\right)$, and $g\left(x^{\prime}\right) \in C^{l}\left(U_{\bar{x}^{\prime}}\right)$.

This result allows one to recover the surfaces of discontinuity of $f(x)$ given the surfaces of discontinuity of $R(p, \alpha ; f)$.

4. Examples show that the Legendre transform $h(\beta)=L g$ of a function $g\left(x^{\prime}\right), x^{\prime} \in \mathbb{R}^{n-1}$, may have domain of definition of dimension less than $n-1$. Since $Q_{f}$ is a union of several varieties of codimension one in $\mathbb{R P}_{n}$ (called components below), the question arises: which of the components of $Q_{f}$ and which of their intersections provide, after applying the generalized Legendre transform defined in [RZ1], parts of $\Gamma=\partial D$ which have codimension one in $\mathbb{R}^{n}$. The answer is given in Theorem 3. This theorem describes $Q_{f}$ in terms of differential geometry of $\Gamma$. Recall that the principal curvatures of a hypersurface $S \subset \mathbb{R}^{n}$, which is the graph of a function $x_{n}=g\left(x^{\prime}\right)$, are the eigenvalues of the matrix $\left(g_{i j}\right) \cdot\left(\delta_{i j}+g_{i} g_{j}\right)^{-1} \cdot\left(1+\sum_{i=1}^{n-1} g_{i}^{2}\right)^{-1 / 2}, g_{i}=\partial g / \partial x_{i}$. One can prove that if $k, k \geq 1$, principal curvatures of a hypersurface $S$ vanish identically, then for every point $P \in S$ there exists an affine $k$-dimensional space $L_{P}$ such that $P \in L_{P} \subset S$.

Theorem 3. (a) Assume that $B_{m}$ is nonempty. Then $m$ principal curvatures of $\widehat{B}_{m}$ vanish identically;

(b) If $k$ principal curvatures of $\Gamma_{1}$ vanish identically, then $\widehat{\Gamma}_{1}$ has codimension $k+1$ in $\mathbb{R P}_{n}$. 
Every point of $\widehat{\Gamma}_{1}$ is a vertex of a cone $K$, which belongs to $\Gamma_{1 j}$, where $\Gamma_{1} \cap \Gamma_{j} \neq \varnothing$. The directrix of $K$ is $(k-1)$-dimensional, and this directrix can be described as follows: Take an arbitrary point $P \in \Gamma_{1}$, and let $L_{k}(P) \subseteq \Gamma_{1}$ be a $k$-dimensional affine space containing $P$, which exists since $k$ principal curvatures of $\Gamma_{1}$ vanish identically. Let $d_{P}:=\left\{(\alpha: p): l_{\alpha p}\right.$ be tangent to $\Gamma_{j}$ at the points of $\left.L_{K}(P) \cap \Gamma_{j}\right\}$, and let $l_{\alpha_{0} p_{0}}$ be tangent to $\Gamma_{1}$ at the point $P$. The vertex of $K$ is the point $\left(\alpha_{0}: p_{0}\right)$. The directrix of $K$ is the set $d_{P}$.

The set $Q_{f}$ is a union of the sets $\widehat{\Gamma}_{i_{1} \cdots i_{k}}, Q_{f}=\bigcup \widehat{\Gamma}_{i_{1} \cdots i_{k}}$ where the union is taken over all combinations of indices $1 \leq i_{k} \leq s$. Theorem 3 gives a recipe to select the components of $Q_{f}$ which yield after the Legendre transform the components of $\Gamma$ of codimension 1, i.e., hypersurfaces $\Gamma_{j}$ which are parts of $\Gamma, \Gamma=\bigcup_{j=1}^{s} \Gamma_{j}$. Note that if a component of $Q_{f}$ has some principal curvatures vanishing identically, then its preimage in $\mathbb{R}^{n}$ has codimension greater than one. Therefore, if one wishes to recover hypersurface-type components of $\Gamma$, then one should apply the Legendre transform to those components of $Q_{f}$, which do not have principal curvatures which vanish identically. Those hypersurfaces $\Gamma_{j}$ which have identically vanishing principal curvatures are reconstructed by applying the generalized Legendre transform, which was introduced in [RZ1], to high-codimension parts of $Q_{f}$ described in Theorem 3(b). The generalized Legendre transform was applied in [Z] to the study of dual varieties in algebraic geometry.

It is well known that the Radon transform may be considered as a Fourier integral operator, so it makes sense to study its action on the wave front set of $f$. In [RZ1] we study a relation of the wave front of $f$ and the set $Q_{f}$.

\section{Proofs OF Theorems 1 AND 2}

We sketch the proofs in the simplest case $m=1, n=2$, but the ideas are similar in the general case.

First we prove that if $D \subset C^{\infty}$ and $f \in C^{\infty}$, then $R(p, \alpha ; f) \in C^{\infty}$ on the set $V_{f}:=\mathbb{R P}_{n} \backslash Q_{f}$. Thus, the singularities of $f$ are in the set $Q_{f}$. Second, we prove that, generically, $Q_{f}$ is a $C^{\infty}$ hypersurface in $\mathbb{R} \mathbb{P}_{n}$ and find the equation of this hypersurface.

Third, we prove that there exists a neighborhood $U$ of a generic point $(\bar{\alpha}: \bar{p})$ and an equation $\zeta(\alpha: p)=0, \nabla \zeta \neq 0$ in $U$, such that (2) holds.

(a) Let us start with the second claim and prove also Theorem 2 for $n \geq 2$. Let $\alpha \cdot x-p=0$ be a tangent plane $l_{\alpha p}$ to $\Gamma$ at a point $\bar{x} \in \Gamma$. Assume that $\alpha_{n} \neq 0$, and write $x_{n}=\beta \cdot x^{\prime}-q, \beta_{i}:=-\alpha_{i} / \alpha_{n}, q:=-p / \alpha_{n}, x^{\prime}=$ $\left(x_{1}, \ldots, x_{n-1}\right)$. Let $x_{n}=g\left(x^{\prime}\right)$ be the equation of $\Gamma$ in a neighborhood $\bar{U}$ of $\bar{x}$, and $\operatorname{det} g_{i j}\left(\bar{x}^{\prime}\right) \neq 0$. Then $\nabla g\left(x^{\prime}\right)=\beta, q=\beta \cdot x^{\prime}-g\left(x^{\prime}\right)$. Thus $q=h(\beta):=$ $L g$. The equation $q=h(\beta)$ is the equation of $Q_{f}$ in the inhomogeneous coordinates $(\beta, q)$. One can prove that if $q \in C^{s}(\bar{U}), s \geq 2$, and $\operatorname{det} g_{i j}\left(\bar{x}^{\prime}\right) \neq$ 0 , then $h \in C^{s}(U)$, where $U$ is a neighborhood of the point $(\bar{\beta}, \bar{q}), \nabla g\left(\bar{x}^{\prime}\right)=$ $\bar{\beta}, \bar{q}=\bar{\beta} \cdot \bar{x}^{\prime}-g\left(\bar{x}^{\prime}\right)$. Since $L$ is involutive, $g=L h$. Theorem 2 is proved.

(b) Let us prove the first claim for $n=2$. Assume that $(\alpha: p) \in V_{f}$, i.e., $l_{\alpha p}$ is not tangential to $\Gamma$. Write $R(p, \alpha ; f)$ as

$$
J:=\int_{a_{1}(q, \beta)}^{a_{2}(q, \beta)} f\left(x_{1}, \beta x_{1}-q\right) d x_{1},
$$


where $a_{i}:=a_{i}(q, \beta)$ are the points of intersection of $l_{\alpha p}$ with $\Gamma$. The integral $J$ is a sum of the integrals over the intervals $\left(a_{1}, b\right),(b, c),\left(c, a_{2}\right)$, where $a_{1}<b<c<a_{2}$ and $b, c$ do not depend on $q, \beta$. Obviously the integral over $(b, c)$ is a $C^{l}$ function of $\beta$ and $q$ if $f \in C^{l}, l \geq 0$. The integrals over $\left(a_{1}, b\right)$ and $\left(c, a_{2}\right)$ are treated similarly.

Let us prove that the integral over $\left(a_{1}, b\right)$ is $C^{l}$ function of $q, \beta$ if $\Gamma, f \in$ $C^{l}, l \geq 2$, and $l_{\alpha p}$ is transversal to $\Gamma$, that is, $\beta \neq g^{\prime}\left(a_{1}\right)$. It is sufficient to prove that $a_{1}(q, \beta) \in C^{l}$. The function $a_{1}(q, \beta)$ is the root of the equation $q=\beta a_{1}-g\left(a_{1}\right)$. By the transversality condition $\beta-g^{\prime}\left(a_{1}\right) \neq 0$. Thus, the implicit function theorem implies that the root $a_{1}(q, \beta) \in C^{l}$ if $g \in C^{l}$. The first claim is proved.

(c) Let us prove the last claim. Let $(\bar{\alpha}: \bar{p}) \in Q_{f}$ and $(\bar{\beta}, \bar{q})$ be the corresponding nonhomogeneous coordinates. For a generic $(\bar{\alpha}: \bar{p})$ the condition $g^{\prime \prime}\left(\bar{x}_{1}\right) \neq 0$ follows from the equation $g^{\prime}\left(\bar{x}_{1}\right)=\bar{\beta}$ and Sard's theorem. We can assume therefore that $g^{\prime \prime}\left(\bar{x}_{1}\right) \neq 0$. Consequently, the point $\bar{x}_{1}$ is a Morsetype (nondegenerate) critical point of the function $z:=\bar{\alpha} \cdot x-\bar{p}$ on $\Gamma \cap \bar{U}$, i.e., of the function $-\bar{\beta} x_{1}+g\left(x_{1}\right)+\bar{q}$. The part of integral (1) taken over the complement to $\bar{U}$ is a $C^{\infty}$-function of $(\alpha: p)$ according to $(b)$. It gives $r_{2}$ in formula (2). By the Morse lemma, there are coordinates $u_{1}, u_{2}$ such that the equation of $\Gamma$ in these coordinates is $u_{1}=0$, the region $D \cap \bar{U}$ is described by the inequality $u_{1} \geq 0$, and $z=u_{1}+u_{2}^{2}$ in $\bar{U}$. To study the singularity of $R(p, \alpha ; f)$, take a curve $\gamma$ which intersects $Q_{f}$ transversally, for instance, $\gamma=\{(\alpha: p): \alpha=\bar{\alpha}\}$. Parameter $p$ gives the position of a point on $\gamma$. On $l_{\bar{\alpha} p}$ one has $\bar{\alpha} \cdot x-p=0$ and $z=\bar{\alpha} \cdot x-\bar{p}$, so $z=p-\bar{p}$ on $l_{\bar{\alpha} p}$. Thus, $z$ can be used as a parameter which determines the position of a point on $\gamma$; therefore, the domain of integration in (1) can be described by the inequality $u_{1} \geq 0$ and the equation $z-u_{1}-u_{2}^{2}=0$. Thus, $z-u_{2}^{2}=u_{1}$, so $-z_{+}^{1 / 2} \leq u_{2} \leq z_{+}^{1 / 2}$ since $z=z_{+} \geq 0$ in the integration region. We have

$$
R(p, \bar{\alpha} ; f)=\int_{l_{\bar{\alpha} p}} f(x) \mu(d x)=\int_{l} f_{1}\left(u_{1}, u_{2}\right) \mu_{1}(d u)=\int_{-z_{+}^{1 / 2}}^{z_{+}^{1 / 2}} f_{2}\left(u_{2}, z_{+}\right) d u_{2},
$$

where $f_{2}\left(u_{2}, z\right)$ is a $C^{\infty}$-function, $l$ is the curve given by the equation $z-u_{1}-$ $u_{2}^{2}=0$ and $u_{1} \geq 0, \mu_{1}(d u)$ comes from $\mu(d x)$ via the Morse lemma change of variables, and the last integral comes after an elimination of $u_{1}$. From this formula one derives (2). Indeed, write $f_{2}\left(u_{2}, z\right)$ as a sum of even $f_{\mathrm{e}}$ and odd $f_{\mathrm{o}}$ functions of $u_{2}, f_{e}\left(u_{2}, z\right) \in C^{\infty}, f_{o}\left(u_{2}, z\right) \in C^{\infty}$. Then the integral

$$
\int_{-z_{+}^{1 / 2}}^{z_{+}^{1 / 2}} f_{\mathrm{e}}\left(u_{2}, z_{+}\right) d u_{2}=z_{+}^{1 / 2} r_{1} \text { and } \int_{-z_{+}^{1 / 2}}^{z_{+}^{1 / 2}} f_{o}\left(u_{2}, z_{+}\right) d u_{2}=0,
$$

where $r_{1} \in C^{\infty}$. The function $r_{2}$ in formula (2) vanishes if $\Gamma$ is strictly convex so that $l_{\bar{\alpha} p}$ intersects $\Gamma$ at two points only.

\section{EXAMPLES}

1. Let $f(x)=1,|x| \leq a, f(x)=0,|x|>a, x \in \mathbb{R}^{n}, n \geq 2, \hat{f}\left(p, \alpha_{0}\right)=$ $2 \sqrt{a^{2}-p^{2}}, \alpha^{0}=\alpha|\alpha|^{-1}$. Thus $p^{2} /|\alpha|^{2}=a^{2}$ is the equation of $Q_{f}$. In $(\beta, q)$ coordinates the equation of $Q_{f}$ is $q= \pm a \sqrt{1+\beta^{2}}, \beta \in \mathbb{R}^{n-1}$. Thus $h(\beta)= \pm a \sqrt{1+\beta^{2}}$. By Theorem 2 the equation $x_{n}=g\left(x^{\prime}\right)$ of the surface 
of discontinuity of $f(x)$ is given by $g\left(x^{\prime}\right)=L h=\mp \sqrt{a^{2}-x^{\prime 2}}$. The equation $x_{n}= \pm \sqrt{a^{2}-x^{\prime 2}}$ defines the sphere $|x|=a$.

2. Let $f(x)=1, b \leq|x| \leq a, f(x)=0,|x|<b$ or $|x|>a, 0<b<a$, $n \geq 2$. Then $\hat{f}\left(p, \alpha^{0}\right)=2 \sqrt{a^{2}-p^{2}}, b \leq p \leq a ; \hat{f}\left(p, \alpha^{0}\right)=2\left(\sqrt{a^{2}-p^{2}}-\right.$ $\left.\sqrt{b^{2}-p^{2}}\right), 0 \leq p \leq b ; \hat{f}\left(p, \alpha^{0}\right)=0, p>a,\left|\alpha^{0}\right|=1$. Thus $p^{2}=|\alpha|^{2} a^{2}$ and $p^{2}=|\alpha|^{2} b^{2}$ are the equations of $Q_{f}$. Taking Legendre's transform yields the surfaces $|x|=a$ and $|x|=b$ of discontinuity of $f(x)$.

3. Consider $f(x)=0$ outside of the region $D$ bounded by $\Gamma$, where $\Gamma$ is the union of the curves $x_{2}=0$ and $x_{2}=x_{1}^{2}-1$, and let $f(x)=1, x \in D$. The $R(p, \alpha ; f)$ is a function whose support is bounded by the curves $q=\beta$, $q=-\beta$ from below, $q=\frac{1}{4} \beta^{2}+1$ in the interval $-2 \leq \beta \leq 2$, and $q=\beta$, $q=-\beta$ for $|\beta| \geq 2$ from above. One can check that on the lines $q= \pm \beta$, $-\infty<\beta<\infty$, the function $R(p, \alpha ; f)$ has a singularity of the type $|z|$ and on the parabola $q=\frac{1}{4} \beta^{2}+1$ it has the singularity of the type $z_{+}^{1 / 2}$. Applying Legendre's transform first to the function $q=\frac{1}{4} \beta^{2}+1,-2 \leq \beta \leq 2$, yields the parabola $x_{2}=x_{1}^{2}-1,-1 \leq x_{1} \leq 1$; and secondly, applying it to the functions $q= \pm \beta$ yields two points $x_{1}= \pm 1, x_{2}=0$. By Theorem 3 , the straignt line joining these two points also belongs to $\Gamma$. Thus $\Gamma$ is recovered.

\section{NUMERICAL ASPECTS}

The RT of $f(x)$ is usually given with an error. Hence, the first numerical problem is to calculate the function $h(\beta)$ which gives the equation of the set $Q_{f}$ of the singularities of RT given the noisy measurements of the RT. The second numerical problem is to calculate $L h=g\left(x^{\prime}\right)$. Calculation of the Legendre transform of a function $h(\beta)$ known with errors is a well-posed problem, at least in the case when $\operatorname{det} g_{i j}\left(x^{\prime}\right) \neq 0$. It is proved in [RSZ] that if a function $g_{\delta}\left(x^{\prime}\right)$ given such that $\left|g_{\delta}\left(x^{\prime}\right)-g\left(x^{\prime}\right)\right|<\delta, g_{\delta}\left(x^{\prime}\right)$ is not necessarily in $C^{2}$ but is continuous, then one can calculate $L g$ with the accuracy $O(\delta)$ as $\delta \rightarrow 0$. This means that a stable method is given in [RSZ] for calculating the Legendre transform of noisy data. See also [R5]. Our result in part 3 of §II has an interesting connection with the envelopes theory $[\mathrm{T}, \mathrm{Zl}]$.

\section{ACKNOWLEDGMENTS}

A. G. Ramm thanks ONR, NSF, and USIEF for support. The research of A. I. Zaslavsky was supported in part by a grant from the Ministry of Science and the "Ma-agara"-special project for absorption of new immigrants, in the Department of Mathematics, Technion.

\section{REFERENCES}

[GGV] I. M. Gelfand, M. I. Graev and N. Ya. Vilenkin, Generalized functions, Integral Geometry and Problems of Representation Theory, vol. 5, Academic Press, New York, 1966.

[N] F. Natterer, The mathematics of computerized tomography, Teubner, Stuttgart, 1986.

[P] V. Palamodov, Some singular problems of tomography, Mathematical Problems of Tomography (I. Gelfand and S. Gindikin, eds.), Amer. Math. Soc., Providence, RI, 1990, pp. 123-150.

[Q] E. Quinto, Tomographic reconstructions from incomplete data-numerical inversion of the exterior Radon transform, Inverse Problems 4 (1988), 867-876.

[R1] A. G. Ramm, Random fields estimation theory, Longman, New York, 1990. 
[R2] Inversion of limited-angle tomographic data, Comp. and Math. with Appl. 22 (1991), 101-112.

[R3] - On numerical differentiation, Izvestiya Vuzov Math. 11 (1968), 131-135.

[R4] Estimates of the derivatives of random functions, J. Math. Anal. Appl. 102 (1984), 244-250; (with T. Miller) 110 (1985), 429-435.

[R5] _ Multidimensional inverse scattering problems, Longman, New York, 1992 (expanded Russian edition will be published by Mir, Moscow, 1993).

[RZ1] A. G. Ramm and A. Zaslavsky, Reconstructing singularities of a function from its Radon transform, Technion, preprint 1992.

[RZ2] - Asymptotic behavior of the Fourier transform of a piecewise smooth function, Technion, preprint 1992.

[RSZ] A. G. Ramm, A. Steinberg and A. Zaslavsky, Stable calculation of the Legendre transform, Technion, J. Math. Anal. Appl. (to appear).

[T] R. Thom, Sur la théorie des enveloppes, J. de Math. Pure Appl. 56 (1962), 177-192.

[Z] A. I. Zaslavsky, Dual varieties and Legendre transforms, submitted.

[Zl] V. A. Zalgaller, The theory of envelopes, Nauka, Moscow, 1975. (Russian)

Mathematics Department, Kansas State University, Manhattan, Kansas 66506-2602

E-mail address: ramm@ksuvm.ksu.edu

Department of Mathematics, Technion-Israel Institute of Technology, 32000 Haifa, ISRAEL

E-mail address: mar9315@technion.technion.ac.il 